\title{
A Sociomaterialidade do Ensino-Aprendizagem em Administração: Perspectivas e desafios
}

\author{
The Sociomateriality of Management Education: Perspectives and Challenges
}

\author{
Cecília Oliveira Bezerra ${ }^{1}$ \\ Eduardo Paes Barreto Davel ${ }^{2}$
}

\section{Resumo}

No ensino-aprendizagem em administração, a dimensão material é desprezada ou preconcebida como mera ferramenta presente na ação humana. Com efeito, evidencia-se uma visão limitada sobre o papel da materialidade como "meio" para a aprendizagem e quase inexistência de compreensão sobre suas questões sociais. Dessa forma, duas carências de pesquisa se apresentam como relevantes: (a) as revisões da produção acadêmica que considerem plenamente a materialidade e (b) a pesquisa construída a partir de uma abordagem sociomaterial. O objetivo desta pesquisa é sistematizar e discutir a produção acadêmica sobre a materialidade do ensino-aprendizagem em administração (EAA), destacando a sociomaterialidade como perspectiva para o avanço de pesquisas futuras. A metodologia baseia-se em uma revisão sistemática da produção acadêmica publicada nas bases nacionais e internacionais. Como resultado, portanto, proporcionamos uma demonstração de como a abordagem sociomaterial descortina novos horizontes para avançar com a pesquisa e impacta em nossos espaços de aprendizagem, no uso e compreensão do potencial dos artefatos e objetos na atividade educacional em instituições de ensino.

Palavras-chave: Ensino-aprendizagem; Materialidade; Sociomaterialidade.

\begin{abstract}
In management education, the material dimension is neglected or pre-conceived as a mere tool present in human action. In fact, there is a limited view on the role of materiality as a 'means' for education and almost no understanding of its social issues. Thus, two research needs are relevant: (a) revisions of academic production that fully consider materiality and (b) research built from a sociomaterial approach. The research goal is to systematize and discuss the academic production on the materiality of management education, highlighting sociomateriality as a perspective allowing advancement of future research. The methodology is based on a systematic review of academic production published on national and international bases. As a result, therefore, we provide a demonstration of how a sociomaterial approach produces new horizons for the advancement of research and impacts our learning spaces, using and understanding the artifacts and objects potentialities in educational activity within educational institutions.
\end{abstract}

Keywords: Teaching-learning; Materiality; Sociomateriality.

Doutoranda em Administração pela Universidade Federal da Bahia (UFBA). Docente do Instituto Federal de Educação, Ciência e Tecnologia Baiano (IFBAIANO). Economics da Universidade Nova de Lisboa (Portugal). 


\section{INTRODUÇÃO}

Aprender é como respirar. Envolve absorver e processar a experiência, expondo e expressando o que é aprendido (KOLB; KOLB, 2005). É a construção física das coisas no mundo que, simultaneamente, constrói o conhecimento do mundo na mente (BARRY; MEISIEK, 2014). No ensino-aprendizagem em administração (EAA), a dimensão material é sistematicamente desprezada, descartada, como se fosse mera ferramenta presente na ação humana (FENWICK, 2016). Estudar a sociomaterialidade no processo educacional envolve uma preocupação em rastrear e reconhecer os efeitos constituídos nas "coisas da educação": estudantes, professores, atividades e espaços de aprendizagem, representações de conhecimento como textos, pedagogia, conteúdo curricular, etc. (FENWICK, 2016).

A materialidade envolve questões como: ambientes físicos e suas configurações espaciais, clima, objetos e suas constituições, ambientes virtuais e interações sociais, etc. (TAYLOR; STATLER, 2014; BARRY; MEISIEK, 2014; PESCHL; FUNDNEIDER, 2012). Todavia, materialidade e sociomaterialidade não são conceitos sinônimos. A sociomaterialidade vai além da materialidade e diz respeito à fisicalidade inseparável entre vida, humanidade e relações sociais (ACTON, 2017, 2018; FENWICK, 2011, 2015). No EAA, a materialidade é pesquisada como "meio" para se desenvolver a aprendizagem, enquanto que suas questões sociais (sociomaterialidade) são raridade. Identificamos somente uma pesquisa que relaciona sociomaterialidade ao EAA de forma ampla e pouco detalhada (FENWICK, 2016).

Não considerar a materialidade no EAA incorre em constranger o entendimento do potencial de elementos materiais na aprendizagem. A falta de entendimento acerca da materialidade dos espaços físicos das escolas, por exemplo, a depender do ponto de vista do professor, pode desconsiderar seus impactos como barreiras ou pequenos impedimentos para as mudanças pedagógicas almejadas, especialmente as focadas em inovação (CHARTERIS; SMARDON; NELSON, 2016). Além disso, não pesquisar a sociomaterialidade no EAA incorre em insistir em um olhar binário sobre os espaços de aprendizagem, os recursos didáticos e o processo educacional. Consequentemente, duas carências de pesquisa se apresentam como marcantes: (a) revisões da produção acadêmica que considerem plenamente a materialidade e (b) pesquisa construída a partir de uma abordagem sociomaterial.

O objetivo desta pesquisa é sistematizar e discutir a produção acadêmica sobre a materialidade do EAA, destacando a sociomaterialidade como abordagem para o avanço de pesquisas futuras. A metodologia de pesquisa envolveu uma revisão sistemática de produções acadêmicas (teses, dissertações, artigos e livros), publicadas prioritariamente nos últimos 20 anos nas bases nacionais e internacionais (SPELL, SciELO, Sage Publications, Routledge, Periódicos CAPES, Library of Congress, Emerald, Jstor, EBSCO, Academy of Management). A escolha dessas bases se deu pela sua relevância e notoriedade no cenário nacional e internacional das pesquisas em administração. Utilizamos como palavras-chave sociomaterialidade, materialidade, ensino-aprendizagem, inovação, administração, sala de aula e espaço de aprendizagem, além de suas correlatas na língua inglesa, envolvendo buscas combinadas entre elas. Ao todo, foram identificadas 4.200 publicações. Finalizada a fase de identificação nas bases, em um primeiro momento, buscamos nos dedicar a analisar, identificar e considerar apenas produções consistentes e coerentes no tratamento da relação entre materialidade, sociomaterialidade e o EAA. Lemos e analisamos os 4.200 títulos e respectivos resumos.

De posse de um número limitado de documentos (apenas duas obras relevantes), dedicamo-nos a um processo de cruzamento de referências na busca da identificação de outras produções relevantes que atendessem ao objetivo proposto. Para esta etapa de cross reference, partimos em duas frentes: (a) os trabalhos sobre materialidade que encontramos nas bases (TAYLOR; STATLER, 2014; BARRY; MEISIEK, 2014) e (b) o trabalho de Acton (2018) sobre sociomaterialidade aplicada ao ensino e aprendizagem. Este trabalho, apesar de não atender ao nosso recorte de pesquisa, se mostrou relevante em sua proximidade com a nossa investigação por resumir um conjunto de tendências advindos do campo da educação. O processo findou quando as obras consultadas começaram a apontar trabalhos já catalogados e nenhuma nova referência emergia como relevante.

A análise do conjunto do material encontrado estruturou-se em quatro eixos: (a) concepções da materialidade no EAA (ensino, aprendizagem e materialidade), (b) âmbitos da materialidade no EAA (espaço, arquitetura, tecnologia e objetos), (c) estímulos da materialidade no EAA (criatividade, inovação, socialização, diversão e emoção) e (d) carências e desafios. O corpus da análise, em todos os seus eixos propostos (concepções, âmbitos, estímulos, carências e desafios), foi construído a partir da análise dos conteúdos encontrados nas obras, quando criteriosamente identificamos os pontos de convergência das investigações e discussões, bem como suas ausências, como as questões de corporeidade que discutiremos no final do artigo.

Ao constatarmos a carência de pesquisas voltadas para a sociomaterialidade no EAA, bem como sua relevância nos estudos de sociomaterialidade em outros campos do conhecimento, desenvolvemos e propomos a abordagem sociomaterial a partir de alguns direcionamentos: (a) definições (sociomaterialidade e ensino-aprendizagem, (b) âmbitos da sociomaterialidade na educação (espaço, arquitetura, tecnologia e objetos) e (c) os impactos da sociomaterialidade na educação (educacional, institucional, espacial e arquitetônico). Os direcionamentos propostos foram estruturados de acordo com o conteúdo analisado nas produções acadêmicas mais relevantes, seja por meio dos exemplos relatados pelos autores ou pelos seus apontamentos para os rumos das pesquisas que abordam a sociomaterialidade aplicada ao contexto educacional. 
Assim, os resultados desta pesquisa contribuem para o avanço nos processos de EAA ao sistematizar conhecimentos que ampliam a sua compreensão para a questão sociomaterial. Ambos importam: matéria e relações entre os humanos e não-humanos (FENWICK, 2016). Se o modo como as pessoas aprendem define o formato de seu desenvolvimento pessoal (KOLB; KOLB, 2005), encontramos na sociomaterialidade uma maneira fundamentalmente distinta de abordar o mundo e a educação. Com a sociomaterialidade, poderemos repensar o que está incorporado nos dispositivos tradicionais de lógica e racionalidade que dominaram a pesquisa em administração (TAYLOR; LADKIN, 2009), provocando assim uma transformação do EAA.

\section{A MATERIALIDADE DO ENSINO-APRENDIZAGEM EM ADMINISTRAÇÃO: BALANÇO E ANÁLISE DOS CONHECIMENTOS ATUAIS}

Quando aplicada ao EAA, a materialidade é tratada, na maioria das pesquisas, de forma implícita (KOLB; KOLB, 2005, 2010; TAYLOR; LADKIN, 2009; CHARTERIS; SMARDON; NELSON, 2018; PESCHL; FUNDNEIDER, 2012; CHIM-MIKI; CAMPOS; ALBUQUERQUE DE MELO, 2019) e, em poucas pesquisas, de forma explícita (TAYLOR; STATLER, 2014; BARRY; MEISIEK, 2014). Sobre a relação entre sociomaterialidade e EAA, somente uma produção foi identificada e aponta algumas possibilidades ao relacionar a sociomaterialidade ao EAA (FENWICK, 2016). Tratase de uma pesquisa que exemplifica aplicações no desenvolvimento de habilidades essenciais em um gestor e que discute questões ligadas à responsabilidade social e à sustentabilidade (FENWICK, 2016).

Nos estudos onde a materialidade no EAA foi tratada de forma explícita, os pesquisadores preocuparam-se apenas em apresentar a materialidade e como ela se relaciona com o processo educacional (TAYLOR; STATLER, 2014; BARRY; MEISIEK, 2014). Nos estudos que tratam a materialidade de forma implícita, os âmbitos e dimensões da materialidade são abordados, mas não há uma preocupação direta em tratar o tema ou desenvolvê-lo de forma robusta (KOLB; KOLB, 2005, 2010; TAYLOR; LADKIN, 2009; CHARTERIS, SMARDON; NELSON, 2018; PESCHL; FUNDNEIDER; 2012; CHIM-MIKI; CAMPOS; ALBUQUERQUE DE MELO, 2019). No único trabalho que foca na sociomaterialidade, encontramos aprofundamento do tema, críticas à abordagem material e novos rumos para as interações sociais no EAA, isto é, interações humanas e não-humanas (FENWICK, 2016).

A precariedade de estudos explícitos sobre a materialidade pode ser atribuída ao fato de que muitos educadores em administração tomam decisões rotineiras sobre mídia, materiais e materialidade em seu ambiente de forma deliberada, apenas com base na prática e sem uma fundamentação científica (TAYLOR; STATLER, 2014). Ainda assim, nestes casos, o material tende a ser desconsiderado ou descartado como meros dispositivos de intenção e ação humana (FENWICK, 2016), mesmo quando a ênfase recai na aprendizagem baseada na prática (TAYLOR; STATLER, 2014; BARRY; MEISIEK, 2014; KOLB; KOLB, 2005, 2010; TAYLOR; LADKIN, 2009; CHARTERIS; SMARDON; NELSON, 2018; PESCHL; FUNDNEIDER, 2012; CHIM-MIKI; CAMPOS; ALBUQUERQUE DE MELO, 2019). Em síntese e de um modo geral, a materialidade é considerada a partir de seus impactos no processo de aprendizagem e como os objetos, seus materiais ou a forma como os espaços se configuram podem promover o alcance de determinados objetivos de aprendizagem (KOLB; KOLB, 2005, 2010; TAYLOR; LADKIN, 2009; CHARTERIS; SMARDON; NELSON, 2018; PESCHL; FUNDNEIDER, 2012; CHIM-MIKI; CAMPOS; ALBUQUERQUE DE MELO, 2019).

No que tange à metodologia utilizada nas produções, cinco trabalhos trazem um tipo de pesquisa puramente teórica, envolvendo, quase sempre, referências de outros campos externos ao da educação gerencial (TAYLOR; STATLER, 2014; BARRY; MEISIEK, 2014; KOLB; KOLB, 2005; TAYLOR; LADKIN, 2009; FENWICK, 2016). Quanto aos quatro estudos empíricos, utilizaram-se análises qualitativas, que ao invés de abordagem diretamente ligada à materialidade, apenas focam em seus elementos (CHARTERIS; SMARDON; NELSON; 2018; PESCHL; FUNDNEIDER, 2012; KOLB; KOLB, 2010; CHIM-MIKI; CAMPOS; ALBUQUERQUE DE MELO, 2019).

\subsection{Principais Concepções na Pesquisa sobre Materialidade no EAA}

Três conceitos são centrais para melhor entender a produção acadêmica sobre materialidade no EAA: ensino, aprendizagem e materialidade. Analisamos a produção acadêmica, buscando entender como esses conceitos centrais eram ancorados em concepções teóricas.

Sobre o conceito de ensino, nas pesquisas a materialidade é vista como um elemento que impacta o processo de desenvolvimento dos professores e o seu desenvolvimento profissional. Algumas preocupações associam o ensino à materialidade e que o ensino constitui um processo pareado com a remodelagem dos espaços de aprendizagem. Ou seja, o desenho material dos espaços orienta-se por princípios pedagógicos que transformam a prática dos próprios professores, alunos e gestores (CHARTERIS; SMARDON; NELSON, 2016). Em contraste, a produção acadêmica critica a concepção predominante do ensino em administração por ser fundamentalmente discursiva: o professor e a exposição de seus saberes. Cada tópico é apresentado em uma sequência linear com pouca repetição. Critica-se, então, o fato da principal estratégia de ensino utilizada ainda ser o contar/narrar, o que leva a apresentar uma tendência a uma abordagem teórica do ensino (KOLB; KOLB, 2005). Assim, o EAA é traduzido pela aquisição de habilidades e conhecimentos que são estranhos para a prática do mundo organizacional e da gestão (TAYLOR; STATLER, 2014). 
Sobre o conceito de aprendizagem, concebe-se como algo que envolve mais do que cognição (TAYLOR; STATLER, 2014), envolvendo também o funcionamento integrado da pessoa em seu pensar, sentir, perceber e se comportar (KOLB; KOLB, 2005). A produção acadêmica apresenta diferentes concepções da aprendizagem. Na perspectiva da aprendizagem experiencial, a aprendizagem é percebida pelo seu processo e não apenas seus resultados, afinal todo aprendizado é um reaprendizado. Assim, aprendizagem experiencial é o processo pelo qual o conhecimento é criado através da transformação de experiência (KOLB; KOLB, 2005). Já na ideia de aprendizagem liderada (CHARTERIS; SMARDON; NELSON, 2016), cabe aos gestores dos espaços de aprendizagem uma prática de liderança que influencie e aprimore a aprendizagem dos estudantes, professores e do próprio líder. Isto reflete em um envolvimento contínuo da aprendizagem com questões relacionadas à equidade e à justiça social (CHARTERIS; SMARDON; NELSON, 2016). Na perspectiva da aprendizagem profunda, a aprendizagem integra totalmente os quatro modos do ciclo da aprendizagem experiencial - experimentar, refletir, pensar e agir (KOLB; KOLB, 2010). Compreende-se, então, que quando abordada a ludicidade na promoção da aprendizagem, o "brincar" se torna um elemento central (KOLB; KOLB, 2010).

O conceito de materialidade é concebido como o contexto material em que a aprendizagem ocorre, a incluir ambientes físicos, layouts, temperatura, qualidade do ar na sala, etc. (TAYLOR; STATLER, 2014, p.3). Relacionar materialidade ao EAA sustenta-se na concepção de que educadores podem, intencionalmente, manipular o nível de envolvimento dos estudantes através da seleção propositada de materiais, a fim de integrá-los em um processo de aprendizagem, e, consequentemente, acarretar no aumento da sua eficácia (TAYLOR; STATLER, 2014). Atualmente, nas estratégias do EAA, as pesquisas identificam pouco uso dessa intencionalidade (TAYLOR; STATLER, 2014). A base material para a aprendizagem é ainda, em sua maioria, composta por textos que legitimam a autoridade científica para a discussão (KOLB; KOLB, 2005), mas que nem sempre fomentam conhecimento da prática. As pesquisas declaram que os materiais possuem linguagem própria, ou seja, recursos próprios, associações e restrições embutidas (KOLB; KOLB, 2005; TAYLOR; STATLER, 2014). Desprezá-los seria, consequentemente, seguir concebendo problemas de negócios sem considerar como estas concepções podem estar sendo afetadas pela materialidade que os produz (TAYLOR; STATLER, 2014).

\section{2 Âmbitos da Materialidade no EAA}

$\mathrm{Na}$ análise da produção acadêmica, buscamos entender como a materialidade era aplicada no EAA. Quatro âmbitos surgiram como centrais para explicar essa aplicação, conforme apresentado no Quadro 1.

Quadro 1 - Âmbitos da Materialidade no EAA

\begin{tabular}{|c|c|c|}
\hline Âmbitos & Categorias & Aplicações e Definições \\
\hline \multirow{11}{*}{ Espaço } & \multirow{3}{*}{ Dimensão física } & Concreto (físico ou virtual), área dedicada ao ensino \\
\hline & & Contêiner \\
\hline & & Espaços criativos \\
\hline & \multirow{3}{*}{ Dimensão conceitual } & Zonas ou regiões de aprendizagem \\
\hline & & Acolhedor e hospitaleiro \\
\hline & & Conversacional \\
\hline & \multirow{5}{*}{ Dimensão habilitante } & Espaços lúdicos de aprendizagem \\
\hline & & Espaços criativos \\
\hline & & Espaços ou ambientes habilitantes \\
\hline & & Ambientes inovadores de aprendizagem \\
\hline & & Estúdios de negócios \\
\hline \multirow{2}{*}{ Arquitetura } & \multirow{2}{*}{ Abordagem arquitetônica } & Aspectos técnicos da arquitetura \\
\hline & & Elementos de design \\
\hline Tecnologia & Recurso da tecnologia da informação e da comunicação & Ferramental \\
\hline \multirow{3}{*}{ Objetos } & \multirow{2}{*}{$\mathrm{Na}$ abordagem material } & Materiais \\
\hline & & Artefatos \\
\hline & $\mathrm{Na}$ abordagem sociomaterial & Elementos de efeito de conexão e atividades \\
\hline
\end{tabular}

Fonte: elaboração própria

\subsubsection{Espaço como âmbito da materialidade no EAA}

O espaço é um dos âmbitos mais explorados na produção acadêmica sobre materialidade no EAA. Identificamos três dimensões sobre o espaço: física, conceitual e habilitante. A dimensão física refere-se a uma visão de concretude dada ao espaço, mesmo se incluir espaços virtuais. Esta concretude aborda sua existência em termos de limites reais. Neste caso, o espaço é visto "como uma área dedicada", seja ela física, virtual ou outra (BARRY; MEISIEK, 
2015). O espaço pode trazer uma percepção associada à ideia de lugar, sendo o lugar um espaço que se tornou corporificado, personalizado e caracterizado através de esforços conscientes e prolongados (BARRY; MEISIEK, 2015). O espaço também é descrito como um contêiner: um local que oferece um conjunto de restrições responsáveis por manter tudo unido.

Algumas pesquisas consideram o espaço como uma área que proporciona uma dinamização e, ao mesmo tempo, contém uma estrutura mínima (PESCHL; FUNDNEIDER, 2012). Quando definidos por espaços criativos (CHIM-MIKI; CAMPOS; ALBUQUERQUE DE MELO, 2019), as pesquisas referem-se aos espaços com configuração estrutural diferenciada da tradicional, capazes de proporcionar interação e comunicação, possibilitando o uso de novas abordagens em suas estruturas fisicamente flexíveis. Os espaços criativos podem contar com o uso da tecnologia como recurso para acessar metodologias ativas de ensino, tais como gamificação (uso de técnicas comuns aos games em situações de não jogo, como situações de aprendizagem) e outros métodos que aliam teoria e prática (CHIM-MIKI; CAMPOS; ALBUQUERQUE DE MELO, 2019 p. 391). Em termos de exemplos do cotidiano, esses espaços tendem a assemelhar-se com espaços de coworking ou ambientes empresariais não convencionais, tais como os da Google (CHIM-MIKI; CAMPOS; ALBUQUERQUE DE MELO, 2019).

Sob a égide da dimensão física, as pesquisas discutem a latente necessidade de mudança no ambiente educacional, em especial na estrutura física das salas de aula, para que possam melhor atender a um novo perfil de educandos e educadores, bem como as demandas pela inovação advindas do mercado (CHIM-MIKI; CAMPOS; ALBUQUERQUE DE MELO, 2019; CHARTERIS; SMARDON; NELSON, 2016; PESCHL; FUNDNEIDER, 2012). Assim, as salas de aula clássicas tendem a dar espaço a salas voltadas para a educação criativa, lúdica e interativa (CHIM-MIKI; CAMPOS; ALBUQUERQUE DE MELO, 2019).

A dimensão conceitual do espaço envolve a ideia de zona ou regiões (KOLB; KOLB, 2005, 2010; PESCHL; FUNDNEIDER, 2012). Consiste em uma concepção da aprendizagem como um processo de locomoção dentre as regiões de aprendizagem, as quais são influenciadas pela posição da pessoa no espaço de aprendizagem em uma lógica metafórica de compartimentação que ocorre em um ambiente psicológico e em que as pessoas experienciam o espaço subjetivamente (KOLB; KOLB, 2005). Seu conceito, associado ao propósito da aprendizagem, não é um processo universal. Trata-se de um "mapa de territórios de aprendizagem", considerado como um quadro de referência de diversas formas de se aprender que podem florescer e interagir dentro de uma estrutura holística que provoca diferentes práticas de aprendizagem (KOLB; KOLB, 2005 p. 200). Na subjetividade do espaço, em seus elementos psicológicos, encontramos a demanda por um espaço hospitaleiro e acolhedor, caracterizado pelo respeito por todos. O espaço precisa ser seguro e solidário, mas também desafiador. $\mathrm{O}$ espaço deve permitir que os estudantes possam ser protagonistas de seu próprio aprendizado, disponibilizando tempo para a prática repetitiva que desenvolve conhecimentos (KOLB; KOLB, 2010).

Outros tipos de espaços incluídos na dimensão conceitual são: o espaço conversacional (KOLB; KOLB, 2005) e o espaço de aprendizagem experiencial. Enquanto o espaço conversacional baseia-se no diálogo, o espaço de aprendizagem experiencial mobiliza forças de atração e repulsão (positivas e negativas) dos dois polos da ação/reflexão e da experiência/conceituação para criar um mapa bidimensional das regiões do espaço de aprendizagem (KOLB; KOLB, 2005). Espaços para boas conversas, como parte do processo educacional, geram oportunidades para refletir e fazer sentido sobre experiências que melhoram a eficácia da aprendizagem experiencial. Ambos requerem clima e/ ou cultura de apoio para que o estudante tenha confiança e se desenvolva ao longo do tempo (KOLB; KOLB, 2005). De forma equilibrada, promovem a relação entre pensamento e sentimento, fala e escuta, liderança e solidariedade, reconhecimento das individualidades e dos grupos, em um processo discursivo e recursivo (KOLB; KOLB, 2005). Encontramos nesta dimensão, portanto, elementos de natureza social, cognitiva, emocional, epistemológica, cultural, organizacional, tecnológica e virtual (PESCHL; FUNDNEIDER, 2012).

A dimensão habilitante refere-se à ideia de espaço associada à sua finalidade ou ao tipo de habilitação à qual ele se propõe: espaços lúdicos de aprendizagem (KOLB; KOLB, 2010), espaços criativos (CHIM-MIKI; CAMPOS; ALBUQUERQUE DE MELO, 2019), espaços habilitantes (PESCHL; FUNDNEIDER, 2012), ou ainda como parte de um ambiente habilitante, tais como os ambientes inovadores de aprendizagem (CHARTERIS; SMARDON; NELSON, 2016) ou os estúdios de negócios (BARRY; MEISIEK, 2015).

Os espaços lúdicos de aprendizagem associam os espaços de aprendizagem (concepção conceitual) ao brincar e à ludicidade como mecanismos de promoção da aprendizagem; uma aprendizagem que ultrapassa as habilidades técnicas envolvidas no jogo, sendo capaz de gerar uma aprendizagem profunda (KOLB; KOLB, 2010). Os espaços criativos, nesta mesma dimensão, estão objetivamente associados à interação, às tecnologias, às funcionalidades, à gamificação e ao ócio criativo. Eles devem permitir o desenvolvimento humano dos estudantes em suas habilidades criativas (CHIM-MIKI; CAMPOS; ALBUQUERQUE DE MELO, 2019). Espaços habilitantes apoiam, possibilitam e facilitam processos de inovação e criação de conhecimento. Baseiam-se na ideia de que habilitar é diferente de gerenciar ou controlar a inovação. Dessa forma, com estruturas facilitadoras do processo de construção do conhecimento, são espaços multidimensionais, tanto na ideia arquitetônica, quanto na conceitual (social, emocional, epistemológico, etc.). São espaços orquestrados de maneira integrada para apoiar as atividades de inovação da melhor forma possível, com foco em inovações radicais em oposição às inovações incrementais (PESCHL; FUNDNEIDER, 2012). 
Os ambientes inovadores de aprendizagem advêm de uma proposta para repensar os espaços escolares. Com origem na Nova Zelândia em 2015, esta proposta faz parte de um movimento para repensar as relações espaciais e reformular a escolaridade de acordo com o discurso da aprendizagem do século XXI (CHARTERIS; SMARDON; NELSON, 2016), visando impactar estudantes, gestores, professores e toda a comunidade acadêmica com suas conexões que vão além dos muros escolares (CHARTERIS; SMARDON; NELSON, 2016). Nesta mesma direção, os estúdios de negócios (BARRY; MEISIEK, 2015) são espaços onde os processos educacionais são interativos e inspiram-se nos estúdios das escolas de arte, adaptando-se às demandas da formação em administração. Estúdios de negócios apresentam forte ênfase na liderança dos participantes por meio de engajamento criativo e prático, com o objetivo de produzir resultados através da reformulação imaginativa de problemas, soluções inovadoras, síntese orientada para um conjunto de habilidades como propõe a "aprendizagem integrativa". Através do "aprender pelo fazer", estúdios de negócios alimentam-se das incertezas dos processos criativos, ao contrário dos tradicionais laboratórios que foram criados para evitar acidentes e situações imprevisíveis (BARRY; MEISIEK, 2015).

\subsubsection{Arquitetura como âmbito da materialidade no EAA}

O âmbito da arquitetura que se aproxima do âmbito do espaço em termos de sua concretude, mas aborda aspectos técnicos espaciais que estruturam e qualificam o espaço. O espaço arquitetônico é um ambiente físico projetado e construído intencionalmente, envolvendo os usuários com suas estruturas físicas concretas (PESCHL; FUNDNEIDER, 2012). Essas estruturas compreendem todos os elementos do espaço e seu contexto, como paredes, móveis, janelas, etc. (PESCHL; FUNDNEIDER, 2012). Assim, a arquitetura contempla dois elementos centrais: a arquitetura em si (em termos de como são construídas suas estruturas) e o design (desenho e ambientação). Estes elementos são profundamente inter-relacionados e não devem ser tratados (ou definidos) como entidades separadas. O design e a arquitetura só fazem sentido se pensados conjuntamente, ao contrário do que muitas vezes ocorre na constituição dos espaços de educação gerencial (PESCHL; FUNDNEIDER, 2012). A arquitetura é vista, então, como um âmbito material apoiador dos processos de criação de espaços habilitantes na busca pela construção do conhecimento e da inovação, porém, ainda assim, não é suficiente mudar a arquitetura para melhorar o EAA. A cultura e atitude da instituição educacional precisam, efetivamente, acompanhar a mudança para conquistar um resultado ativo de modelagem (PESCHL; FUNDNEIDER, 2012). Uma sala de aula como um espaço de educação criativa para o curso de administração precisa ser um ambiente físico totalmente diferente do que conhecemos atualmente (CHIM-MIKI; CAMPOS; ALBUQUERQUE DE MELO, 2019).

O âmbito da arquitetura é pouco explorado na produção acadêmica. A crítica menciona que em muitos contextos que a arquitetura leva, inclusive, a "desabilitar espaços" em vez de habilitar ou de apoiar ativamente os processos de conhecimento e inovação (PESCHL; FUNDNEIDER; 2012). Encontramos somente duas pesquisas que se debruçam sobre estas definições (PESCHL; FUNDNEIDER, 2012; CHIM-MIKI; CAMPOS; ALBUQUERQUE DE MELO, 2019). Mesmo que de forma limitada, caminhos apontam para a importância de alguns elementos da arquitetura e do design nas discussões sobre materialidade e arquitetura em EAA. A proposta envolve a existência de ambientes fechados, o uso de paredes de vidro bem como de paredes lousa, uso de grafites como elemento de decoração, uso de mobiliário modulado e em estilo coworking, mesas coletivas, mesas de reunião, a importância do estudo de cores para estímulo à criatividade e à atenção, visibilidade externa, uso de cores claras com detalhes vivos, a existência de paredes funcionais e a importância da sonorização e da acústica (CHIM-MIKI; CAMPOS; ALBUQUERQUE DE MELO, 2019).

\subsubsection{Tecnologia como âmbito da materialidade no EAA}

A tecnologia envolve o universo da tecnologia da informação e da comunicação, que é visto como elemento essencial para ressignificar e ampliar às possibilidades de atuação nos processos EAA (CHIM-MIKI; CAMPOS; ALBUQUERQUE DE MELO, 2019; PESCHL; FUNDNEIDER; 2012; CHARTERIS; SMARDON; NELSON, 2016; TAYLOR; STATLER, 2014; BARRY; MEISIEK, 2015). A tecnologia e suas ferramentas digitais são capazes de aumentar o interesse e conhecimento dos estudantes do curso de administração (CHIM-MIKI; CAMPOS; ALBUQUERQUE DE MELO, 2019), uma vez que os processos de inovação estão sempre incorporados em um ambiente tecnológico (PESCHL; FUNDNEIDER, 2012).

Compreende-se, então, que as tecnologias digitais medeiam o ensino, bem como a vida dos estudantes (FENWICK, 2016). Professores e estudantes desejam ou precisam de: acesso à internet, computadores com softwares de administração, lousas digitas, acesso a plataformas digitais, acesso a aulas multimídias e equipamentos de conferência (CHIM-MIKI; CAMPOS; ALBUQUERQUE DE MELO, 2019). Dessa forma, apesar de compreendida como âmbito relevante da materialidade, não encontramos detalhamento sobre sua aplicação no EAA de modo mais específico ou detalhado. Para dar a devida ênfase no âmbito da tecnologia, é preciso, portanto, repensar a transformação relacionada à forma como as escolas são concebidas - em termos de ambientes inovadores de aprendizagem - e passar a pensar como deveriam ser se orientadas para uma visão da educação digital do século XXI (CHARTERIS; SMARDON; NELSON, 2016). Em uma abordagem sociomaterial, a tecnologia e seus artefatos emergem 
através da interação com os seres humanos na prática (FENWICK, 2016), tendo esses processos sociomateriais afetando a maneira como os gestores, as gestoras, estudantes, educadores, funcionários e partes interessadas interagem no ambiente on-line (FENWICK, 2016).

\subsubsection{Objetos como âmbito da materialidade no EAA}

Enfocam, nas pesquisas, e de forma central, na materialidade de forma explícita, na preocupação do uso de objetos e de seus materiais (TAYLOR, STATLER, 2014; BARRY, MEISIEK, 2014. Essas pesquisas pontuam que a matéria dos objetos é tratada como algo que provoca diferentes percepções no indivíduo (TAYLOR; STATLER, 2014) e que, em algumas pesquisas, textos e mídias são considerados como elementos diferentes dos objetos.

Mídias servem para comunicar informações, tais como vídeos e textos (TAYLOR; STATLER, 2014). Materiais também são traduzidos por objetos, concebidos como as coisas físicas que são utilizadas na prática do ensino ou coisas que são pedidas para os estudantes produzirem. Assim, embora as pesquisas tratem materiais como relevantes para o EAA, elas destacam que os materiais são frequentemente empregados por educadores da área, sem nenhuma teoria para descrever ou prever os efeitos que geram no processo educacional ou em seus resultados (TAYLOR; STATLER, 2014).

Por conseguinte, mesmo quando há intencionalidade na escolha dos objetos e materiais, em termos científicos, elas se baseiam apenas nos processos educacionais em artes, por se tratar de um campo de prática que tem recebido maior atenção dos teóricos dos estudos organizacionais nos últimos anos (TAYLOR; LADKIN, 2009). O risco de escolher deliberadamente materiais deve-se ao fato de que um objeto pode conter contradições (lógicas e/ou morais), bem como possibilidades não realizadas que não estão limitadas pela lógica ou pelas limitações de nossas vidas atuais (TAYLOR; LADKIN, 2009). O uso de materiais baseados apenas nas experiências educacionais nas artes pode desprezar alguns aspectos da materialidade dos objetos. Para os estudantes de artes, por exemplo, a formação traz um maior foco na estética, enquanto que, para os estudantes de administração, onde o foco é científico, a sua atenção tende a estar mais focada em palavras, símbolos e sinais matemáticos (KOLB; KOLB, 2005).

A importância do uso, produção e presença de objetos nos espaços de aprendizagem explica-se na medida em que os artefatos permitem articular as estruturas de atenção, apreensão e projeção. Tais estruturas sustentam nossas capacidades cognitivas e perceptivas que operam como um campo unificado, ao invés de apenas nossas capacidades intelectuais (TAYLOR; LADKIN, 2009). O artefato opera como uma janela para o poço de coisas, materiais e conhecimentos subconscientes. Neste sentido, então, um artefato pode incorporar contradições e tensões, ao passo que formas mais discursivas não conseguem (TAYLOR; LADKIN, 2009). Destarte, para a abordagem sociomaterial, as coisas devem ser vistas como efeitos de conexões e atividades (FENWICK, 2016).

\subsection{Estímulos da Materialidade no EAA}

A análise da produção acadêmica permitiu identificar como a materialidade impacta o EAA por meio de cinco estímulos: criatividade (CHIM-MIKI; CAMPOS; ALBUQUERQUE DE MELO, 2019; BARRY; MEISIEK, 2015; PESCHL; FUNDNEIDER, 2012), inovação (CHARTERIS; SMARDON; NELSON, 2016; CHIM-MIKI; CAMPOS; ALBUQUERQUE DE MELO, 2019; PESCHL; FUNDNEIDER, 2012), socialização (CHARTERIS ; SMARDON; NELSON, 2016; BARRY; MEISIEK, 2015; KOLB; KOLB, 2010; PESCHL; FUNDNEIDER, 2012), diversão (KOLB; KOLB, 2010; CHIM-MIKI; CAMPOS; ALBUQUERQUE DE MELO, 2019) e emoção (TAYLOR; STATLER, 2014; BARRY; MEISIEK, 2015; KOLB; KOLB, 2005, 2010; TAYLOR; LADKIN, 2009; PESCHL; FUNDNEIDER, 2012). Importante considerar que nesta produção a materialidade envolve possibilidades que descrevem ou determinam contextos materiais no qual a aprendizagem ocorre. Ou seja, no EAA a materialidade é vista como meio, apesar do alerta de que ela precisa ser pensada cientificamente em seu fim (TAYLOR; STATLER, 2014).

Em relação ao primeiro estímulo, a criatividade, ambientes e experiências que consideram a materialidade no EAA sugerem seu impacto positivo no processo criativo. Espaço, arquitetura, tecnologia e objetos representam uma riqueza de estímulos para o desenvolvimento da criatividade e para todo processo cognitivo (CHIM-MIKI; CAMPOS; ALBUQUERQUE DE MELO, 2019; BARRY; MEISIEK, 2015; PESCHL; FUNDNEIDER, 2012). Comunicação e estratégias adequadas, bem como recursos e dispositivos, são capazes de promover interação, transformação, o cultivo da criatividade em si e a polinização de ideias (CHIM-MIKI; CAMPOS; ALBUQUERQUE DE MELO, 2019).

Sobre o segundo estímulo, a inovação, como resultado das ações envolvendo a materialidade no EAA, é também explicada a partir da construção do processo cognitivo. Os processos de criar conhecimento e consequentemente inovar sempre são baseados em processos cognitivos que estão incorporados ou situados em um ambiente físico concreto, social e cultural. (PESCHL; FUNDNEIDER, 2012). Os processos de inovação e de conhecimento estão sempre ancorados em contextos específicos e espaços concretos (PESCHL; FUNDNEIDER, 2012).

O terceiro estímulo refere-se à socialização. Com efeito, materialidade no EAA atua como promotora da socialização do conhecimento. Ambientes de aprendizagem inovadores promovem uma significativa mudança no desempenho dos professores e ainda trazem melhorias significativas no envolvimento e no desempenho dos 
estudantes (CHARTERIS; SMARDON; NELSON, 2016). Este estímulo advém da interação entre o conhecimento e os âmbitos da materialidade (CHARTERIS; SMARDON; NELSON, 2016, BARRY; MEISIEK, 2015, KOLB; KOLB, 2010). Em casos de jogos ou de estúdios de produção coletiva, por exemplo, os estudantes aprendem uns com os outros e interagem na troca. Isso acontece a partir do espaço, dos objetos ou demais elementos de materialidade que são compartilhados (BARRY; MEISIEK, 2015; KOLB; KOLB, 2010).

O quarto estímulo indica que a diversão no EAA provém de três fatores como princípios-chave de um espaço de aprendizagem lúdico propício à aprendizagem profunda: (a) o aprendizado é autêntico com base na criação de regras e condutas em um jogo; (b) o aprendizado adquire significado quando se valoriza, simultaneamente, a experiência e o processo de brincar e (c) a atividade de brincar, baseada em processos de repetição, proporciona amadurecimento e aprofundamento às experiências dos estudantes (KOLB; KOLB, 2010). Neste sentido, o aprendizado não advém dos elementos materiais do jogo ou do elemento lúdico, mas do conhecimento gerado a partir da experiência do fazer junto (BARRY; MEISIEK, 2015; KOLB; KOLB, 2010).

O quinto estímulo, a emoção, sugere que a materialidade gera e/ou permite a mobilização das emoções para a alavancagem/desenvolvimento da aprendizagem (TAYLOR; STATLER, 2014; BARRY; MEISIEK, 2015; KOLB; KOLB, 2005, 2010; TAYLOR, LADKIN, 2009; PESCHL; FUNDNEIDER, 2012). Contudo, a educação gerencial não costuma se preocupar com o nível de energia emocional de uma maneira mais formal, mesmo que os professores estejam sempre se esforçando para alcançar engajamento dos estudantes (TAYLOR; STATLER, 2014). A seleção de materiais e a definição de como eles serão utilizados é feita pelo professor, além de ser uma alavanca poderosa para mobilizar emoções profícuas (TAYLOR; STATLER, 2014). Compreendemos, então, que sentimentos e emoções têm primazia na determinação no nosso aprendizado, já que aprendemos que emoções negativas, como medo e ansiedade, podem bloquear o aprendizado, enquanto sentimentos positivos de atração e interesse podem ser essenciais para o aprendizado (KOLB; KOLB, 2005).

\subsection{Carências e Desafios na Pesquisa sobre Materialidade no EAA}

Durante a análise da produção acadêmica, dois tipos de carências foram identificados. A primeira carência refere-se à concepção limitada da materialidade mobilizada pela produção acadêmica: ausência de diversidade e profundidade de teorias e âmbitos sobre materialidade. Sobre a ausência de diversidade e profundidade de teorias, observa-se que poucos estudos tratam a materialidade como assunto central da sua pesquisa e/ou defende sua relevância como temática (TAYLOR; STATLER, 2014; BARRY; MEISIEK, 2014). Constata-se que na maioria das pesquisas (TAYLOR; STATLER, 2014; BARRY; MEISIEK, 2014; KOLB, KOLB, 2005, 2010; TAYLOR; LADKIN, 2009; CHARTERIS; SMARDON; NELSON, 2018; PESCHL FUNDNEIDER, 2012; CHIM-MIKI; CAMPOS; ALBUQUERQUE DE MELO, 2019) a materialidade é abordada como um recurso ou um meio para se alcançar objetivos educacionais. Em nenhuma pesquisa, a materialidade é considerada como ator ativo do processo educacional. Sobre a ausência de diversidade e profundidade de âmbitos da materialidade, são poucas pesquisas que consideram de forma variada e robusta as questões de espaço, objetos, tecnologias e arquitetura no EAA. E apenas um dos estudos apresentou pesquisa empírica sobre como sua concepção material interfere no EAA.

A segunda carência na produção acadêmica refere-se à ausência de sociomaterialidade. Ou seja, a materialidade não é teoricamente concebida de forma a incluir a dimensão social. Desse modo, sociomaterialidade como conceito é praticamente inexistente nas pesquisas de EAA. Identificamos apenas o trabalho de Fenwick (2016) que aborda a sociomaterialidade no EAA e aponta caminhos para apoiar o administrador no desenvolvimento de habilidades e competências essenciais para o exercício profissional. Indica-se, então, que concepções sociomateriais de práticas podem abrir maneiras úteis de repensar o EAA (FENWICK, 2016).

\section{PERSPECTIVAS EDUCACIONAIS PARA DESENVOLVER A SOCIOMATERIALIDADE NO EAA}

As pesquisas no campo da educação avançaram na forma de conceber as questões materiais com base no conceito de sociomaterialidade. Esta concepção torna explícito o aprendizado e o ensino incorporados, as sinergias entre lugar e pessoas, as relações entre as possibilidades imaginadas, os parâmetros de projeto de infraestrutura e construção, bem como as experiências das pessoas que habitam esses espaços (ACTON, 2018). A partir da análise da produção acadêmica em EAA e da carência identificada por uma teorização mais adequada para fornecer um direcionamento para pesquisas futuras, propomos as pesquisas sobre sociomaterialidade no campo da educação.

\subsection{Definições da sociomaterialidade e do Ensino-Aprendizagem}

A definição de sociomaterialidade foca na fisicalidade intrínseca da vida e das relações sociais, envolvendo uma compressão inseparável das esferas materiais como inseparáveis das sociais (FENWICK, 2011). Isto revela uma ontologia relacional inerente ao conceito de sociomaterialidade (ACTON, 2017) e explicaria, por exemplo, 
como um auditório que coloca todos para ouvir apenas um sujeito inibe a interação entre os demais presentes, restringindo o potencial de trabalhos colaborativos entre os estudantes (BOYS, 2011). Compreende-se, então, que a sociomaterialidade tende a ver todas as coisas conjuntamente, sejam elas humanas ou não humanas, híbridas ou partes, assim como os efeitos de suas conexões e atividades (FENWICK, 2011).

As pesquisas sobre sociomaterialidade sofreram influência de uma variedade de famílias teóricas: a teoria atorrede, estudos sociotécnicos, teoria da complexidade, novos materialismos feministas, geografias pós-estruturais, dentre outros (FENWICK, 2011). Suas raízes teóricas e ontológicas, muito diferentes, desenvolveram tradições particulares em seus distintos campos acadêmicos: filosofia, ciência política, estudos de ciências e tecnologia, geografia humana, física teórica e os estudos organizacionais (FENWICK, 2015). Neste sentido, a materialidade não está consolidada apenas em artefatos/objetos, mas sim distribuída de tal modo que o social, tão quanto os processos físicos, podem ser entendidos como materiais (SØRENSEN, 2009).

Teóricos da educação como Piaget, Dewey e Vigostky conceberam o aprendizado humano na perspectiva de agentes ativos, com enfoque na prática e no fazer (FENWICK, 2011). A sociomaterialidade se apoia em uma visão de aprendizado centrado na importância primordial do pessoal, do material e do social, ou seja, que aceita um mundo mais que humano e investiga sobre o que isso significa para a educação (FENWICK, 2015). Ou seja, tratar a EAA por uma abordagem sociomaterial, ao invés de se apegar a um entendimento (redutivo) da aprendizagem centrada no sujeito e significar o uso do argumento geral de que há muito valor em se considerar uma visão "mais que humana" do mundo (ACTON 2018).

Sob a lente da sociomaterialidade, seres humanos e mais que os seres humanos (objetos, economias, ambientes) constituem coletivamente mundos educacionais (ACTON 2018) ao considerar que as coisas materiais são performativas, agindo juntamente com outros tipos de coisas e forças, para excluir, convidar e regular formas particulares de participação (FENWICK, 2015). Em termos de desempenho, a sociomaterialidade da educação constitui um interconectado entrelaçamento que acarreta na fusão entre espaços, tecnologias, pessoas, ontologias e epistemologias incorporadas, bem como seu propósito e intenção, ao invés de um simples relacionamento causal que flui unilateralmente em qualquer direção do conhecimento (ACTON, 2018). Neste sentido, a definição de ensinoaprendizagem está relacionada aos processos educacionais que se tornam práticas coletivas em relação a significados sociais compartilhados. Como práticas, os processos educacionais estão situados e envolvem o pensar, o fazer e os encontros afetivos. Trata-se, portanto, de um processo de transição, um espaço limiar ou uma jornada, uma negociação de limites ou locais de degola (BOYS, 2011), uma vez que professores e estudantes estão entrelaçados em transições espaciais que visam garantir a "construção ativa e engajada do conhecimento" (ACTON, 2018, p.8).

\section{2 Âmbitos da Sociomaterialidade na Educação}

Destacaremos quatro âmbitos da materialidade (espaço, arquitetura, tecnologia e objetos) para entendê-los sob a lente da sociomaterialidade.

\subsubsection{Espaço sob a lente sociomaterial}

Não é possível dissociar o espaço da aprendizagem já que as questões sociais e espaciais precisam ser conceituadas conjuntamente (MULCAHY; CLEVELAND; ABERTON, 2015) por abordarem questões de associação entre atividades, materiais, espaços e/ou arranjos, mesmo que esta não seja uma correlação direta, transparente e óbvia, entre função e forma. Em termos físicos, embora o espaço de aprendizagem seja compreendido como capaz de causar mudanças comportamentais, a abordagem sociomaterial do espaço muda esta visão na prática, passando o mesmo a ser entendido como reflexo de encontros multifacetados entre os novos espaços, a equipe e os estudantes que os habitam (ACTON, 2017). Os espaços de aprendizagem incluem compromissos problemáticos, colisões e resultados inesperados (BOYS, 2011).

Os espaços de aprendizagem podem ser entendidos a partir de dois eixos: (a) classificação e finalidades e (b) atores envolvidos. Quanto à classificação, espaços são definidos como formais e informais (ACTON, 2018). Espaços formais são aqueles comumente utilizados, tais como salas de aulas com quadro e cadeiras, laboratórios, auditórios e alguns modelos de espaços que visem à aprendizagem colaborativa. Nos espaços informais, encontramos elementos da vida acadêmica (tradicional) e elementos da vida social (ACTON, 2018) que extrapolam ambientes formais, tais como os espaços de convivências, lounges, lachonetes, etc. Em termos de finalidades, os espaços de aprendizagem apresentam um senso comum modernista e funcional, visto ainda como o local onde as coisas acontecem (BOYS, 2011).

Quanto ao eixo de atores envolvidos, a pesquisa espacial visa iluminar, cuidadosamente, as conjunturas, tensões e práticas vividas das relações espaços-sociais (ACTON, 2017). Tanto o espaço, quanto o seu uso, benefícios, propósitos e efeitos são formados através da interação com entidades vivas e não vivas (ACTON, 2017) que envolvem professores, estudantes, arquitetura, tecnologia e objeto (BOYS, 2011; GOURLAY; OLIVER, 2018, ACTON, 2017, 2018; MULCAHY; CLEVELAND; ABERTON, 2015; JAMIELSON, 2008; FENWICK, 2011, 2015). Deste modo, a concepção de espaços de aprendizagem deve ser uma prática conjunta (ACTON, 2018; BOYS, 2011) com sua concepção na 
perspectiva da segurança. Entretanto, isso não significa que irá ser construída uma zona de conforto para o estudante. A segurança, como aspecto social do espaço, representa uma condição necessária para um espaço de aprendizagem (BOYS, 2011). É preciso sentir-se seguro para estar à vontade para se arriscar, bem como ser capaz de lidar com as incertezas. Ao aprender, os estudantes também estão experimentando todas as dificuldades inerentes ao domínio dos limites conceituais. $O$ espaço de aprendizagem deve considerar que o processo de aprender requer uma articulação do que estudantes já sabem com o que eles estão aprendendo (BOYS, 2011). Envolver professores e estudantes na concepção do espaço viabiliza o alcance do comportamento pretendido, pois estudantes deixam de ocupar os espaços somente na lógica dos arquitetos ou designers que os projetaram (JAMIELSON, 2008).

\subsubsection{Arquitetura sob a lente sociomaterial}

Para a sociomaterialidade, prédios e estruturas arquitetônicas não há uma única representação para todos os membros da comunidade educacional e não devem ser construídos visando reconhecimento (BOYS, 2011). A infraestrutura (do espaço físico) deve ser vista como uma assembleia sociomaterial, ou seja, um entrelaçamento que envolve a aprendizagem acadêmica, o ensino, as agendas institucionais, a arquitetura intencional, a tecnologia, os funcionários, os estudantes e os próprios resultados pedagógicos como a forma construída que consistem em uma simbiose ativa de todos os participantes (ACTON, 2017). Interpretações do espaço ocorrem entre o design, sua troca socioespacial e o que as pessoas corporificam - o que veem e são (BOYS, 2011).

Em termos arquitetônicos (design e estrutura), não encontramos pesquisas que enfoque sobre espaços ideais para aprendizagem; a orientação das pesquisas recai no conforto, na atratividade e na flexibilidade dos espaços de aprendizagem. Mesmo em espaços formais, deve-se admitir a presença de elementos intrínsecos a um ambiente informal (BOYS, 2011; JAMIELSON, 2008; ACTON, 2018), uma vez que isso pode viabilizar a realização de atividades diversas, movimentação espacial e conferir ao estudante a possibilidade de controlar mobiliários, objetos, dispositivos tecnológicos e outros materiais (JAMIELSON, 2008). Pela lente sociomaterial, a arquitetura envolve práticas sociais e de aprendizagem a partir de duas premissas essenciais: (a) para desenvolver novos e inovadores espaços de aprendizagem há de se considerar participação e colaboração, e (b) para promover o design no nível da instituição educacional há de se explicar aspectos representativos da aprendizagem institucional (BOYS, 2011).

Nos espaços informais, metáforas se tornam estratégias arquitetônicas apesar de não garantirem interatividade e uso (BOYS, 2011). É possível se criar salas de estudos a partir da metáfora de cafeterias ou de metáforas vinculadas ao coworking. No entanto, isso não significa que a informalidade garantirá a interação que ocorre em sua concepção original. Para escolher recursos arquitetônicos, é necessário refletir se o estudante está sendo ensinado a aprender nos espaços informais pela lógica da criatividade, da colaboração e da interação. Uma reflexão é importante: os aspectos sociais da materialidade foram legitimados nos espaços informais quanto à sua interferência? Por exemplo, se a mudança de um mobiliário ou da estética de um espaço interfere no processo educacional, todavia, é importante que estudantes, professores e gestores compreendam a intencionalidade desta mudança. O que está em questão não é apenas o espaço ser formal ou informal, mas se o ensino e a aprendizagem geram valor e impacto (BOYS, 2011) em termos de resultado acadêmico, desempenho educacional e desenvolvimento pessoal.

Um exemplo de como aproximar a definição arquitetônica do espaço de aprendizagem à abordagem sociomaterial pode ser encontrado em Scott-Webber (2004), que relaciona conhecimento, atividades educacionais e arquitetura/ design. Essa relação estrutura-se em cinco arquétipos de ambientes do conhecimento: (a) ambientes de entrega do conhecimento, (b) ambientes de aplicação do conhecimento, (c) ambientes de criação do conhecimento, (d) ambientes para comunicar o conhecimento e (e) ambientes de para tomada de decisões baseadas no conhecimento (SCOTTWEBBER, 2004). Para a autora, essas novas concepções ajudam a compatibilizar as abordagens pedagógicas com os objetivos de aprendizagem, uma vez que há intencionalidade em sua concepção e configuração. Eles consideram a presença e disposição de elementos materiais a partir da compreensão de como sua presença ou ausência interferem no propósito dos encontros educacionais que lá ocorrerão.

\subsubsection{Tecnologia sob a lente sociomaterial}

$\mathrm{Na}$ abordagem sociomaterial da educação, a tecnologia modifica os aspectos sociais e espaciais da aprendizagem, bem como cria espaços de aprendizagens até então inexistentes (FEWICK, 2011, 2015; GOURLAY; OLIVER, 2018). A tecnologia ressignifica repertórios e se engaja com a potencialidade das coisas, mídias e espaços, ao abrir questões não só relacionadas à aprendizagem on-line e virtual, mas também voltadas às questões sociais e materiais no processo educacional (BOYS, 2011). Neste sentido, objetos e espaços de aprendizagem tomam diferentes percepções a partir das interfaces e do próprio significado da interação homem-máquina (BOYS, 2011). As pesquisas também abordam a possibilidade do desenho de espaços educacionais ricos em tecnologia e interpretados como capazes de enfatizar a flexibilidade no contexto dos espaços físicos. A tecnologia trata ainda aquilo que pode trazer significado e relevância para coisas e espaços (BOYS, 2011). Escolas e universidades, por exemplo, começam a experimentar o uso da internet para ressignificar espaços de aprendizagem, mobilizar e reunir estudantes, melhorar 
a interação com técnicos e professores, criando zonas informais de aprendizagem em suas instituições educacionais (GOURLAY; OLIVER, 2018; BOYS, 2011; ACTON, 2018).

Com as tecnologias digitais cada vez mais difundidas na sociedade e na educação, o uso de recursos tecnológicos (exemplo: celulares) permite interação com outras tecnologias (exemplo: plataformas on-line), mesmo quando se está em movimento, o que marca uma alteração radical na interação social e que reflete nas configurações educacionais (GOURLAY; OLIVER, 2018). Para a sociomaterialidade, um ambiente inovador de aprendizagem precisa ser rico em tecnologia com acesso facilitado a todos (professores, estudantes, gestores e equipe de apoio), seja nos espaços formais ou informais de aprendizagem (ACTON, 2018). A tecnologia representa o potencial habilitador dos objetos, redes de relacionamento e espaços, e se abre para as práticas de ensino e aprendizagem em caminhos de trabalho não vistos antes, e, além disso, também se apresentam como irrealizáveis, devido aos espaços e instalações existentes (GOURLAY; OLIVER, 2018).

\subsubsection{Objetos sob a lente sociomaterial}

A provisão de um espaço de aprendizagem que ofereça suporte material a uma experiência de aprendizagem transformadora encontra-se no âmago dos novos projetos de espaço de aprendizado universitário (ACTON, 2018). $\mathrm{Na}$ lente sociomaterial, as matérias importam e agem como decretos, conjuntamente com outros tipos de coisas, e forçam a excluir, convidar e regular formas particulares de participação (FENWICK, 2011). A sociomaterialidade busca compreender (a) as relações sociais e materiais diárias, (b) porque a matéria importa e (c) como desmascarar as abstrações que nos impedem de perceber a microdinâmica que move as práticas cotidianas (FENWICK, 2015). Objetos, eventos, identidades e conhecimentos são entendidos a partir das relações sociais e materiais (FENWICK, 2015).

As coisas importam, mas não como objetos reificados em suas propriedades. Objetos (ou artefatos) têm um efeito dinâmico de materialização que emergem das reuniões (físicas e sociais) e agem em determinados emaranhados da prática diária local (SØRENSEN, 2009). O objeto estabelece uma intensa relação com outros âmbitos da sociomaterialidade: (a) na relação espaço-objeto, em que os objetos deixam de ser apenas elementos contidos em um espaço; (b) na relação arquitetura-objeto, em que o design envolve mais do que a seleção de objetos de mobiliário e ambientação; e (c) na relação tecnologia-objeto, em que o objeto não é apenas um dispositivo de acesso à tecnologia. (ACTON, 2017, 2018; GOURLAY; OLIVER, 2018; BOYS, 2011; FENWICK, 2011, 2015). Em espaços formais ou informais de aprendizagem, objetos se engajam no estabelecimento de relações sociais, seja por meio da sua representatividade em uma tomada, por exemplo, no acesso à internet para leitura de um texto on-line ou como cadeiras móveis e agrupáveis que viabilizam atividades e práticas pedagógicas. Objetos atuam em termos de conforto e flexibilidade para seu uso ou viabilizam a socialização entre estudantes, professores e equipe de suporte. Os objetos são, de fato, elementos não-humanos, mas não por isso deixam de ser elementos sociais (GOURLAY; OLIVER, 2018; FENWICK, 2011, 2015). As configurações materiais são mais do que um pano de fundo para a ação humana. Elas enredam e são partícipes dos acontecimentos mais que humano e, ao mesmo tempo, sociais e corporais (ACTON, 2018).

\subsection{Impactos da sociomaterialidade na Educação}

Os principais impactos da sociomaterialidade nas práticas educacionais podem ser pensadas a partir de quatro eixos: impacto educacional, impacto espacial, impacto arquitetônico, impacto institucional.

O impacto educacional é gerado na esfera do aprender e do ensinar. É um tipo de impacto oriundo da adoção de métodos para reconhecer e rastrear as diversas lutas, negociações e acomodações. Seus efeitos constituem as "coisas da educação": estudantes; professores; atividades e espaços de aprendizagem; representações de conhecimento como textos, pedagogia, conteúdo curricular, etc. (ACTON, 2018). O foco encontra-se nas relações entre as "coisas" e como elas se influenciam e se alteram quando interagem, seja abrindo ou excluindo novas possibilidades de ensino e de aprendizagem (FENWICK, 2015). Com a sociomaterialidade, entendemos melhor como a prática educacional é afetada pelos materiais e questionamos da tendência geral de subestimar os materiais como meros instrumentos para melhorar o desempenho educacional (SØRENSEN, 2009).

Incorporar uma abordagem sociomaterial crítica à educação é perturbar, não apenas modelos de práticas e suposições predominantes sobre a aprendizagem, mas também fundamentos e investimentos cruciais. As abordagens sociomateriais oferecem uma maneira de traçar os pilares das relações entre humano e não humano que trazem realidades particulares à prática e aprendizado, destacando as oportunidades e pontos de entrada para a mudança (FENWICK, 2015). A sociomaterialidade na educação busca traçar como essas relações trabalham, ou seja, como forças humanas e não humanas agem umas sobre as outras mutuamente, transformando suas características e atividades, estabilizando-as ou potencializando-as (FENWICK, 2015).

O impacto institucional refere-se à esfera política, hierárquica e identitária da instituição de ensino. Encontramos na sociomaterialidade diferentes ênfases e posições sobre o papel político dos materiais na constituição de formas particulares da vida cotidiana e do aprendizado (FENWICK, 2015). As abordagens sociomateriais oferecem 
abordagens significativas para a compreensão das relações de poder e da política que constituem a aprendizagem. A sociomaterialidade não se reduz a um dispositivo analítico para separar as maneiras pelas quais as relações intra e interinstitucionais são montadas, mas também aponta maneiras afirmativas de intervir, perturbar ou ampliar esses relacionamentos (FENWICK, 2015).

Na década de 1960, os espaços sociais nas universidades eram valorizados e apontavam aspectos que traziam imponência ao status da instituição de ensino e atribuía valor à identidade organizacional (BOYS, 2011). De lá para cá, esta visão permanece predominantes em duas lógicas essenciais, porém incongruentes com o princípio da sociomaterialidade, como: (a) o design das instituições de ensino está mais preocupado com a representação que isso traduz sobre sua qualidade e (b) sua interpretação e representação assumidas não estão conectadas com nenhuma teoria ligada à estrutura (no sentindo arquitetônico). A estrutura arquitetônica deve pautar-se no processo educacional, em que a prioridade recai em como a aprendizagem ocorre e o desempenho é alcançado (BOYS, 2011).

Espaços de aprendizagem melhorados exigem mais do que os aspectos estéticos. Torna-se essencial se desenvolver uma cultura colaborativa e, consequentemente, superar a estrutura antiga, baseada em estilos de especialista, representadas pelos prédios exclusivos para atividades administrativas ou recortado por áreas do conhecimento (BOYS, 2011). Nas instituições de ensino, a lente sociomaterial estimula uma meta de longo prazo: a criação de uma organização de aprendizagem capaz de desenhar uma instituição representada pelo seu coletivo e envolvendo diferentes expertises (JAMIELSON, 2008). Isso impacta as estruturas hierárquicas, a gestão orçamentária e a comunicação com todos os públicos e com a comunidade (BOYS, 2011) local. Nesse processo, o corpo acadêmico deve permanecer concentrado nos estudantes, na conectividade, na flexibilidade e no desempenho digital (ACTON, 2018).

Já o impacto espacial da sociomaterialidade considera o envolvimento de professores e estudantes nas questões espaciais. Esse envolvimento impacta as práticas e resultados do processo educacional (ACTON, 2018), uma vez que o espaço é o local onde a aprendizagem ocorre e representa a interseção entre o que sabemos e fazemos, e o que não sabemos (BOYS, 2011). O contexto espacial não garante uma renovação das práticas pedagógicas (ACTON, 2018), mas tratar os espaços de aprendizagem de forma não binária é reconhecer as relações sociais inerentes à prática educacional (FENWICK, 2011, 2015; ACTON, 2018).

Em termos físicos, as interações socioeducacionais pretendidas deverão ser evidenciadas também no impacto arquitetônico, pois, além disso, é no espaço que nos envolvemos e testamos nossas experiências cerebrais e corporificadas, tanto no sentido pessoal quando coletivo (BOYS, 2011). Em salas de aulas formais, por exemplo, é comum que o ambiente físico molde as expectativas, como também já molda o tamanho das turmas e permite, impede ou exclui certas possibilidades de ação (JAMIELSON, 2008). Pensar o espaço pela lente sociomaterial, é pensar em um contexto social que envolve matéria, pessoas, histórias, vivências, conhecimentos prévios e conhecimentos pretendidos (FENWICK, 2015).

O impacto arquitetônico refere-se às questões sobre espaço físico e suas configurações, como as configurações dos espaços formais e informais de aprendizagem. Envolve também aspectos relacionados à ambientação em termos de flexibilização, acolhimento, presença da tecnologia e estímulo à criatividade, muitas vezes representados por seu mobiliário e demais objetos de ambientação (BOYS, 2011; ACTON, 2018). O impacto arquitetônico inclui um repensar de projetos para instituições de ensino, tornando-se um novo desafio para os arquitetos e designers de interiores, uma vez que as regras que orientam a construção de espaços de aprendizagem foram criadas há mais de quatro décadas (BOYS, 2011; ACTON, 2018). Assim, professores e estudantes precisam participar da construção destes projetos para se evitar repetição de coisas generalistas sobre a arquitetura como flexibilidade e conforto (BOYS, 2011).

A partir da sociomaterialidade, o projeto arquitetônico e seu design dependem de convenções e premissas, ou seja, daquilo que os participantes trazem para o espaço, a atividade e o contexto, não sendo possível separar as coisas da educação da concepção do projeto. Portanto, os projetos arquitetônicos devem envolver pensar salas de aula em diferentes formatos e layouts, na intenção de que atendam ao que se pretende ensinar e a aprendizagem almejada. Priorizar o conhecimento requer projetos de salas de aulas variadas, tais como: auditório, estúdios, laboratórios ou até espaços de coworking, (CHIM-MIKI; CAMPOS; ALBUQUERQUE DE MELO, 2019). Requer também considerar configurações e mobiliário de cafés, lounges ou hubs (ACTON, 2018; FENWICK, 2011, 2015). A ocupação é sempre um encontro, seja com objetos, seja com espaços e outros. Seus participantes trazem consigo o que já sabem sobre o espaço (ou suas expectativas sobre como ele será ou deveria ser), seja em relação à atividade a ser realizada ou seu contexto (BOYS, 2011). Um layout não proíbe por si só a colaboração, mas pode inibir. Do mesmo modo, ter um espaço flexível com mobiliário móvel pode facilitar a colaboração (ACTON, 2017; SCOTT-WEBBER, 2004), mas não garante que os estudantes se sintam automaticamente empoderados para modificação e mobilização do mobiliário, buscando diferentes disposições, nem que, ainda, consigam trabalhar a partir de uma lógica de colaboração (BOYS, 2011).

\section{DISCUSSÃO}

A partir de uma revisão sistemática da produção acadêmica, investigamos como a materialidade e a sociomaterialidade são tratadas no âmbito do EAA e estruturamos os resultados encontrados em diferentes eixos compreensivos: (a) concepções da materialidade no EAA (ensino, aprendizagem e materialidade), (b) âmbitos da 
materialidade no EAA (espaço, arquitetura, tecnologia e objetos), (c) estímulos da materialidade no EAA (criatividade, inovação, socialização, diversão e emoção) e (d) carências e desafios, a fim de demonstrar pontos de encontros nos estudos já realizados, bem como suas lacunas. Pela inexistência de estudos substanciais sobre a sociomaterialidade na EAA, buscamos compreender como este tema é tratado pela educação, sistematizamos conteúdos relevantes e discutimos os principais fundamentos sobre sociomaterialidade (definições, âmbitos e impactos).

As repercussões desses resultados para pesquisas futuras podem ser elaboradas e discutidas a partir de dois eixos: teórico-conceituais e práticos. Assim, as repercussões_teórico-conceituais envolvem três reflexões: (a) a ponte que a sociomaterialidade estabelece com preocupações contemporâneas no EAA, (b) a renovação crítica dos espaços educacionais nas escolas de administração, e (c) a ausência de questões relacionadas ao corpo e corporeidade.

No que se refere aos impactos teórico-conceituais, destacamos que desde 1990 estamos vivendo a era da nova percepção acerca dos efeitos da matéria na construção social. $O$ debate envolve pesquisas que destacam as questões da agência de elementos não-humanos (MOURA; BISPO, 2020) que performam e geram impactos na dinâmica e nos resultados organizacionais (LEONARDI, 2011), especialmente quando associadas às questões da tecnologia (ORLIKOWSKI, 2007, 2009; ORLIKOWSKI; SCOTT, 2008). No campo da administração, a sociomaterialidade estabelece uma ponte com preocupações contemporâneas no EAA. Os estudos organizacionais promoveram uma discussão sobre a constituição da matéria por atores sociais do ambiente organizacional, incluindo preocupações com ética e políticas (INTRONA; HAYES, 2011). São quase 20 anos de discussões pautadas em teorias que discorrem sobre a agência não-humana e suas influências (LEONARDI, 2011). Entretanto, ainda não conseguimos mobilizar essas contribuições para avançar a pesquisa sobre EAA (FENWICK, 2015). Afinal, se toda materialidade é social (ORLIKOWISKI, 2007), como desconsiderá-la na perspectiva educacional?

Discutir as questões da materialidade em uma abordagem social aplicadas ao EAA envolve também embarcarmos nas suas imbricações. "Imbricar significa organizar elementos distintos em padrões sobrepostos para que funcionem de forma interdependente" (LEONARDI, 2011, p.5). Imbricações sociomateriais no contexto do EAA requerem uma compreensão do funcionamento dos processos, práticas e espaços educacionais de modo interdependente. Isso estimula o fortalecimento da intencionalidade e o respeito pelas questões diversas da interação sinergética (LEONARDI, 2011) das "coisas" da educação. Ou seja, as imbricações sociomateriais são heterogêneas, não imaginadas e não intencionais (INTRONA; HAYES, 2011), mas existem, interferem e devem ser consideradas como elementos presentes nos processos educacionais.

A pesquisa em EAA pode enfocar como renovação crítica dos espaços educacionais nas escolas de administração para se beneficiar da abordagem sociomaterial. Dessa forma, como o contexto formativo pode representar a condição básica para a ação, reforçando as restrições cognitivas e práticas, dando direção e significado? Como este contexto pode promover uma gama de oportunidades para empreender a ação (INTRONA; HAYES, 2011)? Como a materialidade pode fornecer várias possibilidades, seria possível que um artefato produzisse vários resultados educacionais (LEONARDI, 2011)? Quais são esses resultados? Qual é o sentido de continuarmos a estruturar salas de aulas que não traduzem as necessidades e desejos de professores e estudantes? Qual sentido de pensarmos espaços educacionais que não estimulem a colaboração e a interação entre os elementos presentes, a partir das diversas camadas que lá estão sobrepostas? Responder essas perguntas pode ser o ponto de partida para pesquisas futuras no campo do EEA.

É latente, portanto, a necessidade de transformação no ambiente educacional, em especial na estrutura física das salas de aula. Espaços de aprendizagem, incluindo-se as salas de aula, precisam ser repensados nas instituições de ensino em administração para que possam melhor atender às demandas de professores e estudantes. Será que a sociomaterialidade dessas instituições atuais garantem plenamente e adequadamente a formação do(a) gestor(a) para atuar na contemporaneidade? As qualidades necessárias para que gestores liderem com os desafios da contemporaneidade são desenvolvidas no processo educacional e sociomaterial realizado em instituições de ensino? O que se percebe é que a pesquisa em EAA precisa se debruçar sobre essas questões para avançar em seu poder de explicação de como a sociomaterialidade pode ser uma chave importante para repensar a formação em administração.

Em todas as produções acadêmicas sobre materialidade e sociomaterialidade no EAA, o corpo e a corporeidade não são mencionados e nem incluídos como categorias significativas. $\mathrm{Na}$ análise da produção acadêmica, apenas uma das obras sinalizou a corporificação do aprendizado em uma abordagem indireta e superficial (BOYS, 2011). O processo educacional acontece no corpo, portanto, este é parte inseparável do contexto sociomaterial (HOCKING et al., 2001; SINCLAIR, 2005; SWAN, 2005). A consideração sistemática da corporeidade pode abrir novas frentes de pesquisa para melhor entender como o corpo e a sociomaterialidade afetam o processo educacional. Com efeito, pesquisas futuras podem relacionar a corporeidade com a sociomaterialidade para refletir sobre suas potencialidades e desafios no EAA.

As repercussões práticas desta pesquisa referem-se à transformação dos espaços de aprendizagem, às dinâmicas de aprendizagem e ao trabalho de professores e gestores de organizações educacionais. Embora as posições sejam diferentes sobre a compreensão da natureza da agência dentro das redes sociomateriais, a sua potencialidade é essencial. Pela lente sociomaterial, espaços de aprendizagem podem assumir novas configurações, arquiteturas e funcionalidades. Professores podem identificar novas dinâmicas associadas à presença da tecnologia ou ainda intensificar sua interação para além da presencialidade e da sincronicidade da sala de aula tradicional. 
Gestores das organizações educacionais podem melhor compreender que sua tarefa envolve mais que um processo prescritivo e racional. Sua gestão deve ser processual e dinâmica, considerando o que é produzido, reproduzido e modificado cotidianamente (MOURA; BISPO, 2021).

\section{CONCLUSÃO}

O objetivo desta pesquisa foi sistematizar e discutir a produção acadêmica sobre a materialidade do EAA, destacando a sociomaterialidade como abordagem para o avanço de pesquisas futuras. Para alcançar esse objetivo, analisamos a produção acadêmica e desenvolvemos categorias explicativas (concepções, âmbitos, estímulos, carências, desafios), tanto no campo do EAA quanto da educação. Dessa forma, demonstramos como a abordagem sociomaterial fomenta novos horizontes para pesquisar e praticar as dinâmicas educacionais em administração.

A contribuição dos resultados desta pesquisa para o avanço do conhecimento em EAA é variada. Primeiro, o artigo destaca e consolida uma produção acadêmica rarefeita em EAA: a materialidade do processo educacional. Segundo, o artigo organiza e fornece um encaminhamento para pesquisas futuras fundamentarem seus estudos a partir das produções acadêmicas no campo da educação sobre a sociomaterialidade. Assim, pesquisadores dispõem de uma base teórico-conceitual mais estruturada e mapeada para conduzirem suas pesquisas. E por fim, a terceira dimensão da contribuição ocorre quando o artigo provoca uma reflexão sobre potenciais transformações da prática educacional em instituições de ensino.

\section{REFERÊNCIAS}

ACTON, R. E. Place-people-practice-process: using sociomateriality in university physical spaces research. Educational Philosophy and Theory, [s. I.], v. 49, n. 14, p. 1441-1451, 2017.

ACTON, R. E. Innovative learning spaces in higher education: perception, pedagogic practice and place. 2018. Tese. (Doutorado em Filosofia) - James Cook University, Austrália, 2018.

BARRY, D.; MEISIEK, S. Discovering the business studio. Journal of Management Education, [s. I.], v. 39, n. 1, p. 153-175, 2014.

BOYS, J. Towards creative learning spaces: re-thinking the architecture of post-compulsory education. London: Routledge, 2011.

CHARTERIS, J.; SMARDON, D.; NELSON, E. Innovative learning environments and discourses of leadership: is physical change out of step with pedagogical development? Journal of Educational Leadership, Policy \& Practice, [s. I.], v. 31, n. 1/2, p. 33-47, 2016.

CHIM-MIKI, A. F.; CAMPOS, D. B.; MELO, L. S. A. de. Definindo espaços de educação criativa no ensino superior de administração através de mecanismos de cocriação de valor. Administração: Ensino e Pesquisa, [s. l.], v. 20, n. 2, p. 362-401, 2019.

FENWICK, T. Emerging approaches in educational research: tracing the socio-material. London: Routledge, 2011.

FENWICK, T. Sociomateriality and learning: a critical approach. In: SCOTT, D.; HARGREAVES, E. (ed.) The SAGE handbook of learning. London: Sage Publications, 2015. p. 83-93.

FENWICK, T. What matters in sociomateriality: towards a critical posthuman pedagogy for management education and educators. In: STEYAERT, C.; BEYES, T.; PARKER, M. (ed.). The routledge companion to reinventing management education. London: Routlege, 2016. p. 249-263.

GOURLAY, L.; OLIVER, M. Student engagement in digital university: sociomaterial assembleges. Londron: Routledge, 2018.

HOCKING, B.; HASKELL, J.; LINDS, W. (ed.). Unfolding bodymind: exploring possibility through education. Brandon: Foundation for Educational Renewal, 2001.

INTRONA, L. D.; HAYES, N. On sociomaterial imbrications: what plagiarism detection systems reveal and why it matters. Information and Organization, [s. I.], v. 21, n. 2, p. 107-122, 2011. 
JAMIELSON, P. Creating new generation learning environments on the university campus. [S. I.]: WB Research Press, 2008.

KOLB, A. Y.; KOLB, D. A. Learning styles and learning spaces: enhancing experiential learning in higher education. Academy of Management Learning and Education, [s. I.], v. 4, n. 2, p. 193-212, 2005.

KOLB, A. Y.; KOLB, D. A. Learning to play, playing to learn: a case study of a ludic learning space. Journal of Organizational Change Management, [s. I.], v. 23, n. 1, p. 26-50, 2010.

LEONARDI, P. M. When flexible routines meet flexible technologies: affordance, constraint, and the imbrication of human and material agencies. Management Information Systems Quarterly, [s. I.], v. 35, n. 1, p. 147-167, 2011.

MOURA, E. O. de; BISPO, M. de S. Compreendendo a prática da gestão escolar pela perspectiva da sociomaterialidade. Organizações \& Sociedade, [s. I.], v. 28, n. 96, p. 135-163, 2021.

MOURA, E. O. DE; BISPO, M. DE S. Sociomateriality: theories, methodology, and practice. Canadian Journal of Administrative Sciences, [s. I.], v. 37, n. 3, p. 350-365, 2020.

MULCAHY, D.; CLEVELAND, B.; ABERTON, H. Learning spaces and pedagogic change: envisioned, enacted and experienced. Pedagogy, Culture and Society, [s. I.], v. 23, n. 4, p. 575-595, 2015. ORLIKOWSKI, W. J; SCOTT, S. V. Sociomateriality: challenging the separation of technology, work and organization. The Academy of Management Annals, [s. I.], v. 2, n. 1, p. 433-474, 2008.

ORLIKOWSKI, W. J. Sociomaterial practices: exploring technology at work. Organization Studies, [s. I.], v. 28, n. 9, p. 1435-1448, 2007.

ORLIKOWSKI, W. J. The sociomateriality of organisational life: considering technology in management research. Cambridge Journal of Economics, [s. I.], v. 34, n. 1, p. 125-141, 2009.

PESCHL, M.; FUNDNEIDER, T. Spaces enabling game-changing and sustaining innovations: why space matters for knowledge creation and innovation. Journal of Organisation Transformation \& Social Change, [s. I.], v. 9, n. 1, p. 41-61, 2012.

SCOTT-WEBBER, L. In Sync: environmental behavior research and the design of learning spaces. Ann Arbor: Society for Gollege and University Planning, 2004.

SINCLAIR, A. Body and management pedagogy. Gender, Work and Organization, [s. I.], v. 12, n. 1, p. 89-104, 2005.

SORENSEN, E. The materiality of learning: technology and knowledge in educational practice. New York: Cambridge University Press, 2009.

SWAN, E. On bodies, rhinestones, and pleasures: women teaching managers. Management Learning, [s. I.], v. 36, n. 3, p. 317-333, 2005.

TAYLOR, S. S.; LADKIN, D. Understanding arts-based methods in managerial development. Academy of Management Learning and Education, [s. I.], v. 8, n. 1, p. 55-69, 2009.

TAYLOR, S. S.; STATLER, M. Material matters: increasing emotional engagement in learning. Journal of Management Education, [s. I.], v. 38, n. 4, p. 586-607, 2014. 


\section{Contato:}

Cecília Oliveira Bezerra

E-mail: cissabezerra@gmail.com

Eduardo Davel

E-mail: davel.eduardo@gmail.com

Submetido em: 03/10/2020

Revisado em: 21/06/2021

Aprovado em: 04/08/2021 